\title{
Effect of resins on the salt spray resistance and wet adhesion of two component waterborne polyurethane coating
}

https://doi.org/10.1515/epoly-2019-0046

Received March 05, 2019; accepted March 29, 2019.

\begin{abstract}
Effects of the type, chemical structure and $\mathrm{NCO} / \mathrm{OH}$ of resins on wet adhesion and salt spray resistance of two component waterborne polyurethane coating were studied in this paper. The content of functional groups in resins and films were analysed by areas of their FT-IR absorption peak to study the relationship with anticorrosive performance of coatings. Coatings based on resins with more $-\mathrm{OH}$ tend to have stronger wet adhesion and salt spray resistance because resins with more -OH have more possibilities to react with -NCO to form a compact film with a higher crosslinking degree. The wet adhesion of coatings after $8 \mathrm{~h}$ of immersion deteriorated from level 1 to level 5 with the increase of $\mathrm{NCO} / \mathrm{OH}$ due to the decrease of - $\mathrm{OH}$ in coatings to link with substrates. And excess - NCO tend to react with $\mathrm{H}_{2} \mathrm{O}$ to generate $\mathrm{CO}_{2}$ to form cracked physical bubbles, which would weaken the wet adhesion.
\end{abstract}

Keywords: resin; two component waterborne polyurethane coating; salt spray resistance; wet adhesion; hydroxyl group

\section{Introduction}

Metal corrosion is chemical or electrochemical reaction between metals and the environment medium such as $\mathrm{H}_{2} \mathrm{O}, \mathrm{O}_{2}$ and $\mathrm{NaCl}$. To apply anticorrosive coatings (1), such as epoxy (2,3), alkyd resin (4), or polyurethane coating, on the surface of metals is an efficient and economic method

\footnotetext{
* Corresponding author: Qianwei Xu, Tongji University, School of Materials Science and Engineering, No. 4800 Caoan Road, Jiading, Shanghai, Shanghai 201804, China, email:xu_qw@tongji.edu.cn Qingli Lu and Wanwen Shan, Tongji University, Department of Polymeric Materials, School of Materials Science and Engineering, China
}

Steven Zhu and Robin Pang, Covestro Polymers China Co Ltd, Polymer Research and Development Center, China of reducing corrosion. And PU is widely used as the film former of coating due to its good adhesion and resistance to corrosion.

Traditional solvent based PU coating would bring about volatile organic polymerization compounds (VOCs) during coating manufacture and application, which seriously threatens environmental or public safety. Two component waterborne polyurethane ( $2 \mathrm{~K}$ WPU) coating (5) provides a good solution for eliminating VOCs and has been paid progressive attention in coating industry.

There are many reviews in the literature dealing with metal corrosion by WPU coatings based on various resins. Xu et al. (6) prepared a high molecular weight polyacrylate dispersion using the synthesized polyacrylate as macromolecular emulsifier by emulsion polymerization. The WPU coating based on the obtained polyacrylate with low VOCs level showed good wet adhesion and chemical resistance. Huang et al. (7) prepared and characterized electroactive waterborne polyurethane (EWPU) containing conjugated segments of electroactive amino-capped aniline trimer unit. And the protection efficiency $\left(\mathrm{P}_{\mathrm{EF}} \%\right)$ of EWPU (93.96\%) was higher than that of non-electroactive waterborne polyurethane (NEWPU) coating (18.20\%) based on electrochemical measurements in $3.5 \mathrm{wt} \% \mathrm{NaCl}$ electrolyte, which indicated enhanced corrosion protection effects of EWPU on cold-rolled steel (CRS). The enhanced corrosion protection ability of the EWPU coating could be interpreted as that the redox catalytic capabilities of aniline trimer units existed in EWPU induce the formation of passive metal oxide layers on the CRS electrode. Kiosidou (8) used salt spray to test a newly developed polyurethane based marine antifouling coating, and the experimental formulation exhibited superior anticorrosion performance overall compared with acrylic based system.

In addition, it is vital to establish the relationship between resins and anticorrosive properties of coatings such as salt spray resistance (9) and wet adhesion $(10,11)$. $\mathrm{Wu}$ et al. (12) found that the wet adhesion of epoxy coatings was significantly influenced by the epoxide 
content, and excess epoxide groups were highly likely to favor the formation of stronger adhesive bonding at coating-metal interface. Meis et al. (13) concluded that the novel aliphatic resin coatings show better wet adhesion compared to aromatic resin coatings according to the investigation of different epoxy-amine coatings on the adhesion performance on aluminium substrates. Coatings mainly rely on resins and reinforcement to realize superior anticorrosive properties. And the effect of resins in $2 \mathrm{~K}$ WPU coating on the wet adhesion and salt spray resistance cannot be ignored. But most of works (14-17) in recent years have focused on the nano particles, such as nano $\mathrm{ZnO}$ and graphene oxide, to improve anticorrosive performance of $2 \mathrm{~K}$ WPU coating. And the relationship among resins, salt spray resistance, and wet adhesion of $2 \mathrm{~K}$ WPU coating is still not clear.

In this paper, in order to improve anticorrosive performance by selecting resins and optimization of $\mathrm{NCO} / \mathrm{OH}$ during preparation of coatings, $2 \mathrm{~K}$ WPU coating on carbon steel surfaces with $\mathrm{TiO}_{2}$ as the barrier and pigment were fabricated. The crosslinking degree, salt spray resistance, and wet adhesion of $2 \mathrm{~K}$ WPU coating were investigated. The chemical structure of resins and coatings were characterized by Fourier transform infrared spectroscopy (FT-IR), and the content of functional groups were creatively analyzed according to the area of their absorption peak. The scanning electron microscopy (SEM) was used to investigate the micro morphology of coatings. These works intend to study the effect of resins such as their type, chemical structure, and $\mathrm{NCO} / \mathrm{OH}$ in $2 \mathrm{~K}$ WPU coating on corrosion protection and establish the relationship among the chemical structure of resins, salt spray resistance, and wet adhesion of coatings.

\section{Experimental}

\subsection{Materials}

Waterborne hydroxyl-functional polyacrylic polyol (AR70, AR42, AR46, AR27 and AR01), polyester polyol (ES 66) and polycarbonate polyol (CA50), and polyisocyanurate based on HDI trimmers (CU55) were donated by Covestro. Hydroxyl content of selected polyacrylic polyols is different, as shown in Table 1. Wetting and dispersing agent BYK190, surface active agent BYK349, flatting agent BYK381, defoaming agent BYK022 and BYK093 were purchased from BYK-Chemie GmbH. Rheological agent RM8W was purchased from Rohm and Haas. Surface active agent AD01 were purchased from Air Products.
Table 1: Details about - $\mathrm{OH}$ content of resins and - $\mathrm{NCO}$ content of hardener.

\begin{tabular}{lccc}
\hline Component & No. & $\begin{array}{c}\text { Main functional } \\
\text { group }\end{array}$ & $\begin{array}{c}\text { Content of -OH } \\
\text { or -NCO (wt\%) }\end{array}$ \\
\hline Polyacrylic polyol & AR01 & $-\mathrm{OH}$ & 4.0 \\
Polyacrylic polyol & AR70 & $-\mathrm{OH}$ & 3.7 \\
Polyacrylic polyol & AR46 & $-\mathrm{OH}$ & 3.5 \\
Polyacrylic polyol & AR42 & $-\mathrm{OH}$ & 3.0 \\
Polyacrylic polyol & AR27 & $-\mathrm{OH}$ & 2.0 \\
Polyester polyol & ES66 & $-\mathrm{OH}$ & 4.1 \\
Polycarbonate polyol & $\mathrm{CA50}$ & $-\mathrm{OH}$ & 4.0 \\
Polyisocyanurate & $\mathrm{CU} 55$ & $-\mathrm{NCO}$ & 20.0 \\
\hline
\end{tabular}

Auxiliary solvent PGDA were purchased from DOW. $\mathrm{TiO}_{2}$ (R706) was purchased from DuPont. Acetone and alcohol were supplied by Shanghai Ling Feng Chemical Reagent. All the materials above mentioned were used without further purification.

\subsection{Instruments and measurements}

FTIR-ATR spectrum of the samples was measured using FTIR spectrophotometer (EQUINOX 55, German). The micro morphologies inside WPU coating was characterized via field-emission SEM (Quanta200, USA).

\subsection{Preparation of $2 K$ WPU coating}

Firstly, the pigment paste of coatings was prepared by mixing $250.0 \mathrm{~g}$ R706, $10.0 \mathrm{~g}$ AD01, $25.0 \mathrm{~g}$ BYK190, $2.0 \mathrm{~g}$ BYK349, $3.0 \mathrm{~g}$ BYK022, and 2.0 g RM8W with some deionized water at $2500 \mathrm{rpm}$ in a plastic container at $25^{\circ} \mathrm{C}$ for $15 \mathrm{~min}$. After grinding, the $36.9 \mathrm{~g}$ reaction mixture, $0.5 \mathrm{~g}$ BYK381 and $1.0 \mathrm{~g}$ BYK093 were added into $61.6 \mathrm{~g}$ waterborne hydroxyl-functional resin to mix at $2500 \mathrm{rpm}$ for $20 \mathrm{~min}$ and then placed for $12 \mathrm{~h}$ to let out air bubbles. In the next step, 2K WPU coating was prepared by mixing the reaction solution with the hardener CU55 in the presence of PGDA by hand for $3 \mathrm{~min}$. Its viscosity can be adjusted by adding deionized water. The calculation of dosage of resins or hardener is based on the content of - $\mathrm{OH}$ or - $\mathrm{NCO}$ provided by suppliers. And $\mathrm{M}_{\mathrm{w}}$ of selected resins are nearly equal.

\subsection{Application of 2K WPU coating on steel substrates}

The carbon steel panels with dimensions of $15 \mathrm{~cm} \times 7.5 \mathrm{~cm} \times 0.1 \mathrm{~cm}$ were used as metal substrates. Prior 
to coating application, panels were polished with $\mathrm{SiC}$ papers up to 400 grit, and cleaned by ethanol, followed by drying at $25^{\circ} \mathrm{C}$ for use. And the obtained $2 \mathrm{~K}$ WPU coating was applied on the cleaned panels with film applicator, followed by drying in a vacuum oven at $60^{\circ} \mathrm{C}$ for $16 \mathrm{~h}$ to remove possible residual solvents. These samples were allowed to cure at room temperature for 7 days. After curing, these samples with dry film thickness of $55 \pm 5 \mu \mathrm{m}$ were chosen for test.

\subsection{Crosslinking degree test}

Films encased by a sieve were soaked in acetone for $24 \mathrm{~h}$, and they were dried at $60^{\circ} \mathrm{C}$ for $1 \mathrm{~h}$. Their crosslinking degree can be calculated based on the decrement of weight using following equations:

$$
q=\frac{m_{p}}{m_{p}+m_{r} \times n_{r}+m_{h} \times n_{h}} \times 100 \%
$$

Equation 1 describes the ratio of pigment in the film where $m_{p}$ is weight of pigment, $m_{r}$ is weight of resins, $n_{r}$ is solid content of resins, $m_{h}$ is weight of hardener, and $n_{h}$ is solid content of hardener.

$$
c=\left[1-\frac{m_{d}}{m_{f} \times(1-q)}\right] \times 100 \%
$$

Equation 2 describes the crosslinking degree of films where $m_{f}$ is weight of films and $m_{d}$ is decrement of weight of films.

\subsection{Wet adhesion test}

Edges of all the samples were sealed by water-proof tape before immersion. These panels were immersed in the water at $40^{\circ} \mathrm{C}$ for several hours and then tested immediately after being picked up and wiping water off the coating surface. Cross cut test for films was used to classify the wet adhesion of different coatings on substrates according to ISO 2409:2013. The wet adhesion was characterized by evaluating the intact coating area. Stronger wet adhesion can be marked lower level.

\subsection{Salt spray test}

The salt spray test was carried out in a DE2006-7 cyclic corrosion tester cabinet. The corrosive nature of the chamber specified by ASTMB 117 is a continuous spraying with a $3.5 \mathrm{wt} \%$ solution of $\mathrm{NaCl}$ at $35 \pm 1^{\circ} \mathrm{C}$. The back side and edges of steel panels with $\mathrm{x}$-cut paints (according to EN ISO 17872:2007) were protected with a special pressure adhesive tape (TESA Tape, USA) and epoxy. And the coated samples were placed inside the salt spray chamber angled at $45^{\circ}$. Specimen surfaces and the rusting degree were monitored and rated by visual examination of these panels after $600 \mathrm{~h}$.

\section{Results and discussion}

\subsection{Analysis of functional groups content via FT-IR spectra}

Figure 1a shows the FT-IR spectrum of different resins including waterborne polyacrylic polyols (AR70, AR42, AR46, AR27 and AR01) and waterborne hydroxylfunctional polyester polyol (ES66) and polycarbonate polyol (CA50). These spectrums show the absorption
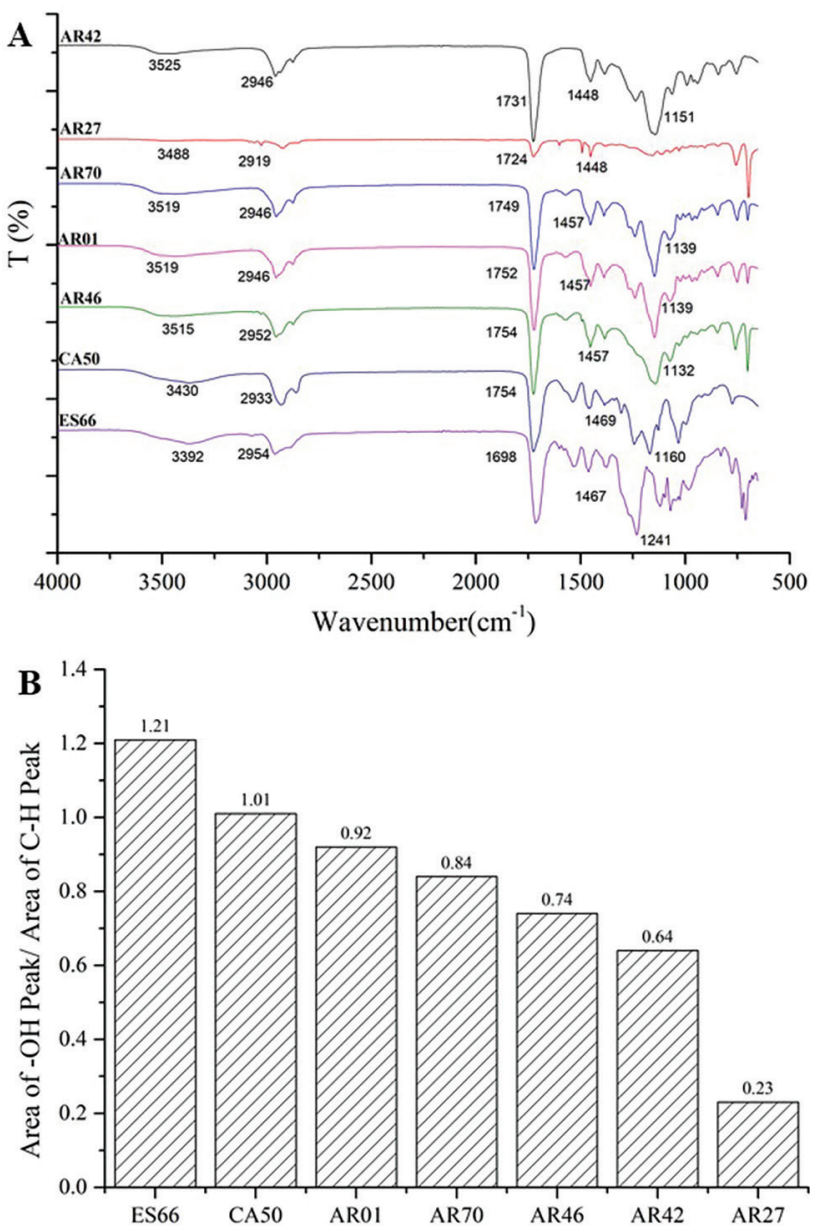

Figure 1: FTIR spectrum (a) of different resins and area of $-\mathrm{OH}$ absorption peak (b) in FT-IR spectrum of different resins. 
peaks at $3500 \mathrm{~cm}^{-1}$ (O-H stretching vibration), $2950 \mathrm{~cm}^{-1}$ $\left(-\mathrm{CH}_{2}\right.$ and $-\mathrm{CH}_{3}$ stretching vibration), $1750 \mathrm{~cm}^{-1}(\mathrm{C}=\mathrm{O}$ stretching vibration) and $1140 \mathrm{~cm}^{-1}(\mathrm{C}-\mathrm{O}-\mathrm{C}$ stretching vibration), respectively.

The area of absorption peaks for some functional groups was calculated to reflect their content in resins or coatings based on the internal standard $\mathrm{CH}$ absorption peak. And the hydroxyl content in resins reflected by the area of $-\mathrm{OH}$ absorption peak was presented in Figure $1 \mathrm{~b}$. The resins can be sorted according to their hydroxyl content based on the analysis of area of absorption peak: $\mathrm{ES} 66>\mathrm{CA} 50>$ AR01 $>$ AR70 > AR46 > AR42 > AR27. As waterborne hydroxyl-functional polyester polyol, ES66 has the highest hydroxyl content among these resins. For same waterborne polyacrylic polyol, the content of -OH in AR61 is the highest, while the content of $-\mathrm{OH}$ in AR27 is the lowest. The different hydroxyl content in polyacrylic polyols indicates that they are ideal materials to study the effect of hydroxyl content in resins on the salt spray resistance and wet adhesion of coatings.

As shown in Figure 2, the hydroxyl content in 2K WPU coating decreased with the increase of $\mathrm{NCO} / \mathrm{OH}$ when the ratio is smaller than 1.6, and then kept stable according to the analysis of area of - $\mathrm{OH}$ absorption peak. This result demonstrated that the proper increase of - $\mathrm{NCO}$ can bring about the consumption of - $\mathrm{OH}$, but excess - $\mathrm{NCO}$ is not available for the reaction with $-\mathrm{OH}$. The absorption peak of - $\mathrm{OH}$ in the case of excess - $\mathrm{NCO}$ means that - $\mathrm{OH}$ in coatings cannot be completely consumed by -NCO. The result can be explained by that chain segments are more difficult to move in $2 \mathrm{~K}$ WPU coating with the chemical reaction going on, and it is difficult for the rest of - $\mathrm{OH}$ to react with -NCO.

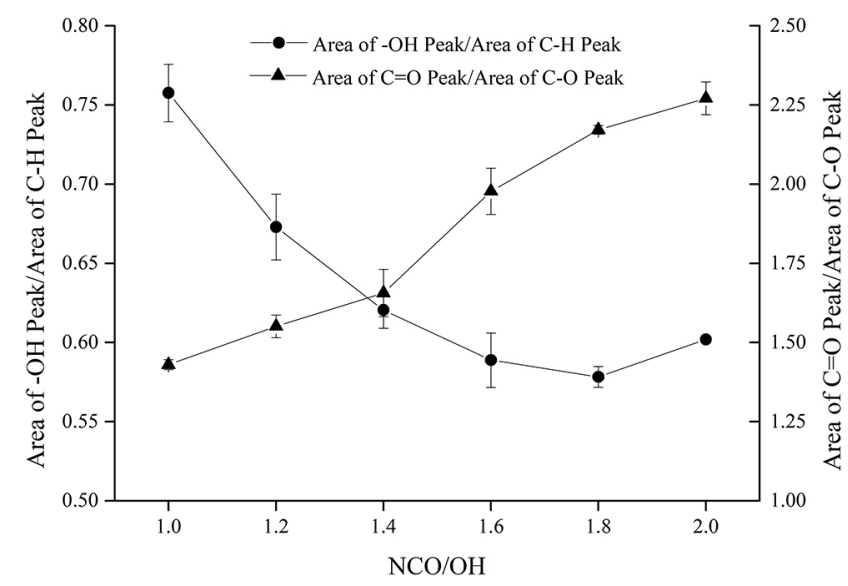

Figure 2: Area of $-\mathrm{OH}$ absorption peak and $\mathrm{C}=\mathrm{O} / \mathrm{C}-\mathrm{O}$ ratio in $2 \mathrm{~K}$ WPU coating with increase of $\mathrm{NCO} / \mathrm{OH}$.
No absorption peak of -NCO in coatings detected by FTIR indicates that the excess -NCO was completely used up. In general, $2 \mathrm{~K}$ WPU coating mainly needs the reaction between $-\mathrm{NCO}$ and $-\mathrm{OH}$ at the molar ratio of 1:1 to form a compact PU film, as expressed in Scheme 1. According to the integration for absorption peaks of $\mathrm{C}=\mathrm{O}$ and $\mathrm{C}-\mathrm{O}$ in FTIR spectra of $2 \mathrm{~K}$ WPU coating with different $\mathrm{NCO} / \mathrm{OH}$, the result was listed in Figure 2. The $\mathrm{C}=\mathrm{O} / \mathrm{C}-\mathrm{O}$ ratio in coatings raises with the increase of $\mathrm{NCO} / \mathrm{OH}$, which means excess - $\mathrm{NCO}$ reacted with $\mathrm{H}_{2} \mathrm{O}$ to generate $\mathrm{CO}_{2}$ and urea without $\mathrm{C}-\mathrm{O}$, as shown in Scheme 1. When $\mathrm{NCO} / \mathrm{OH}$ is smaller than 1.6 , the reaction with $-\mathrm{NCO}$ prevailed because $-\mathrm{OH}$ is easier to react with $-\mathrm{NCO}$ than $\mathrm{H}_{2} \mathrm{O}$. When $\mathrm{NCO} / \mathrm{OH}$ is larger than 1.6, the reaction to generate $\mathrm{CO}_{2}$ and urea dominated due to less $-\mathrm{OH}$ in coatings. The generated $\mathrm{CO}_{2}$ would gather to form physical bubbles in coatings. And these bubbles would remain or break up inside the film while the motion of $\mathrm{PU}$ macromolecule is restricted, which is regarded as a micro defect in $2 \mathrm{~K}$ WPU film.

\subsection{Effect of resins on the crosslinking degree of $2 \mathrm{~K}$ WPU coating}

The crosslinking degree of $2 \mathrm{~K}$ WPU coating based on different resins was presented in Figure 3a. The coating based on polyester polyol ES66 and polycarbonate polyol CA50 showed a higher crosslinking degree than polyacrylic polyol AR01. And resins can be sorted according to the crosslinking degree of $2 \mathrm{~K}$ WPU coating: ES66 > CA50 $>$ AR01 > AR70 > AR46 > AR42 > AR27. The performance in crosslinking degree of coatings is consistent with the hydroxyl content in resins. Resins with more hydroxyl groups reacted and crosslinked with polyisocyanurate as

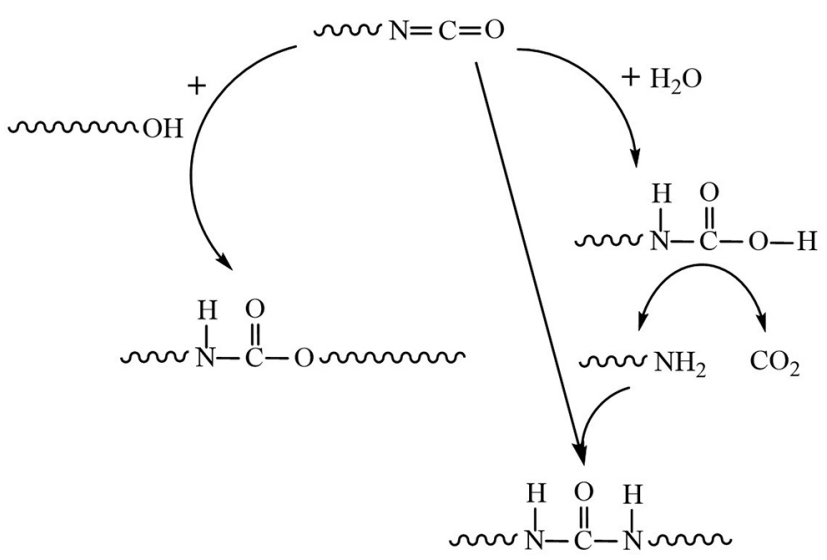

Scheme 1: Reaction mechanism between excess - $\mathrm{NCO}$ and $-\mathrm{OH}$ or $\mathrm{H}_{2} \mathrm{O}$. 


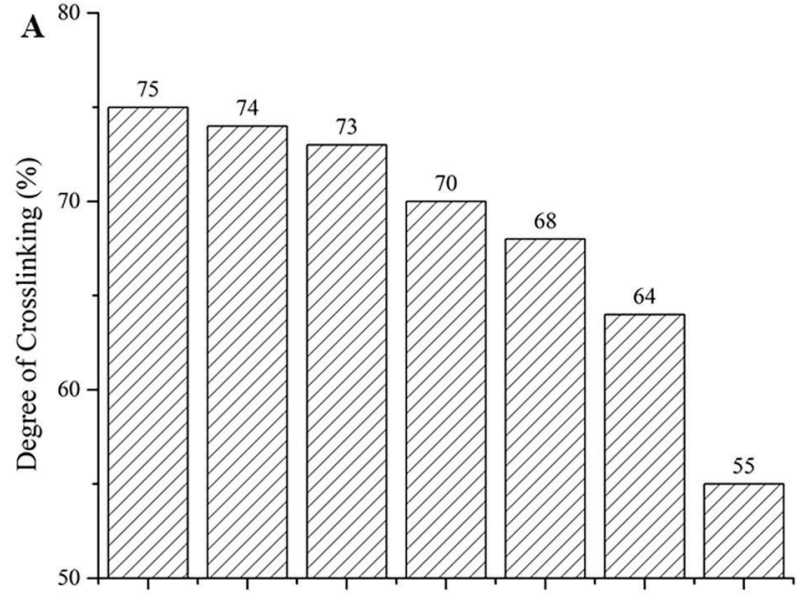

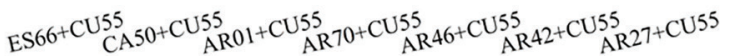
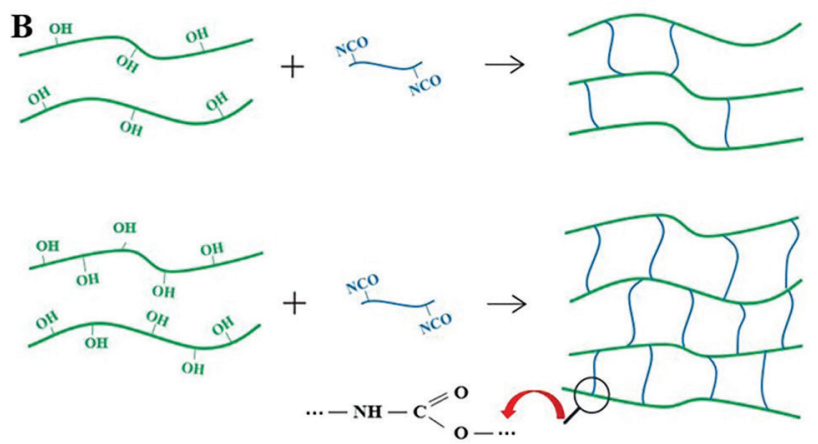

Figure 3: The crosslinking degree (a) of $2 \mathrm{~K}$ WPU coating based on different resins and main reaction mechanism (b) between resins with different hydroxyl content and polyisocyanurate.

shown in Figure 3b, which contributed to the formation of a compact and tight PU film.

\subsection{Morphology analysis of $2 \mathrm{~K}$ WPU coating via SEM}

SEM image of micro morphology inside 2K WPU coating based on different resins was presented in Figure 4. The micro structure of 2K WPU coating based on AR01, ES66, and CA50 seem compact and tight, and enough PU resin wrapped around some pigment particles. The micro structure of 2K WPU coating based on AR27 seem loose by comparison, and many pigment particles are gathering and exposed in coating for lack of enough PU resin. This difference in micro structure is related to the hydroxyl content of resins and crosslinking degree of coatings. The coating based on resin with more $-\mathrm{OH}$ tends to have a high crosslinking degree, and then to form enough PU to wrap around pigment particles. And the formation of compact and tight PU film containing pigment particles is beneficial to shield from corrosive medium, such as $\mathrm{H}_{2} \mathrm{O}, \mathrm{O}_{2}, \mathrm{Cl}, \mathrm{Na}^{+}$and so on. The loose micro structure of WPU coating based on AR27 is caused by low hydroxyl content of resin and low crosslinking degree of coating, which may lead to the decrease of anticorrosive performance.

\subsection{Effect of resins on the salt spray resistance}

\subsubsection{Effect of hydroxyl content in resins on the salt spray resistance}

The corrosion degree of coatings for polyacrylic polyols with different hydroxyl content can be sorted as follows: AR01 > AR70 > AR46 > AR42 > AR27, as shown in Figure $5 \mathrm{a}$. Obviously, the salt spray resistance of $2 \mathrm{~K}$ WPU coating is related with the hydroxyl content in resins. For polyacrylic polyol, more hydroxyl group in resins means more possibilities to react with -NCO. As a result, PU film will become more compact because of its higher crosslinking degree, and its salt spray resistance will be stronger. Consequently, much rust and blister can be seen from the coating based on AR27 with least hydroxyl group at the $\mathrm{OH} / \mathrm{CH}$ ratio of 0.23 , which exhibited almost no barrier to rust accumulation. And the coating based on AR01 with most hydroxyl group at the $\mathrm{OH} / \mathrm{CH}$ of 0.92 showed the best appearance among these samples.

The salt spray resistance of $2 \mathrm{~K}$ WPU coating always depends on the compactness and wet adhesion of the film. In most cases, the compactness of coating relies on the content of groups to react in resins. The more $-\mathrm{OH}$ in resins, the more possibilities for resins to react and crosslink with the polyisocyanurate. Therefore, the compactness of PU film will be improved by selecting high hydroxyl content resins. As for the wet adhesion, it is not only the last barrier of coating to prevent the surface of substrates from corrosive medium, but also the crucial factor to realize its salt spray resistance.

\subsubsection{Effect of different types of resins on the salt spray resistance}

Image of 2K WPU coating on the substrate for different resins including polyacrylic polyol, polyester polyol, and polycarbonate polyol after salt spray for $600 \mathrm{~h}$ were presented in Figure 5b. Little rust and blister on the surface of coatings based on ES66 and CA50 shows the superiority of polyester and polycarbonate polyol on the 

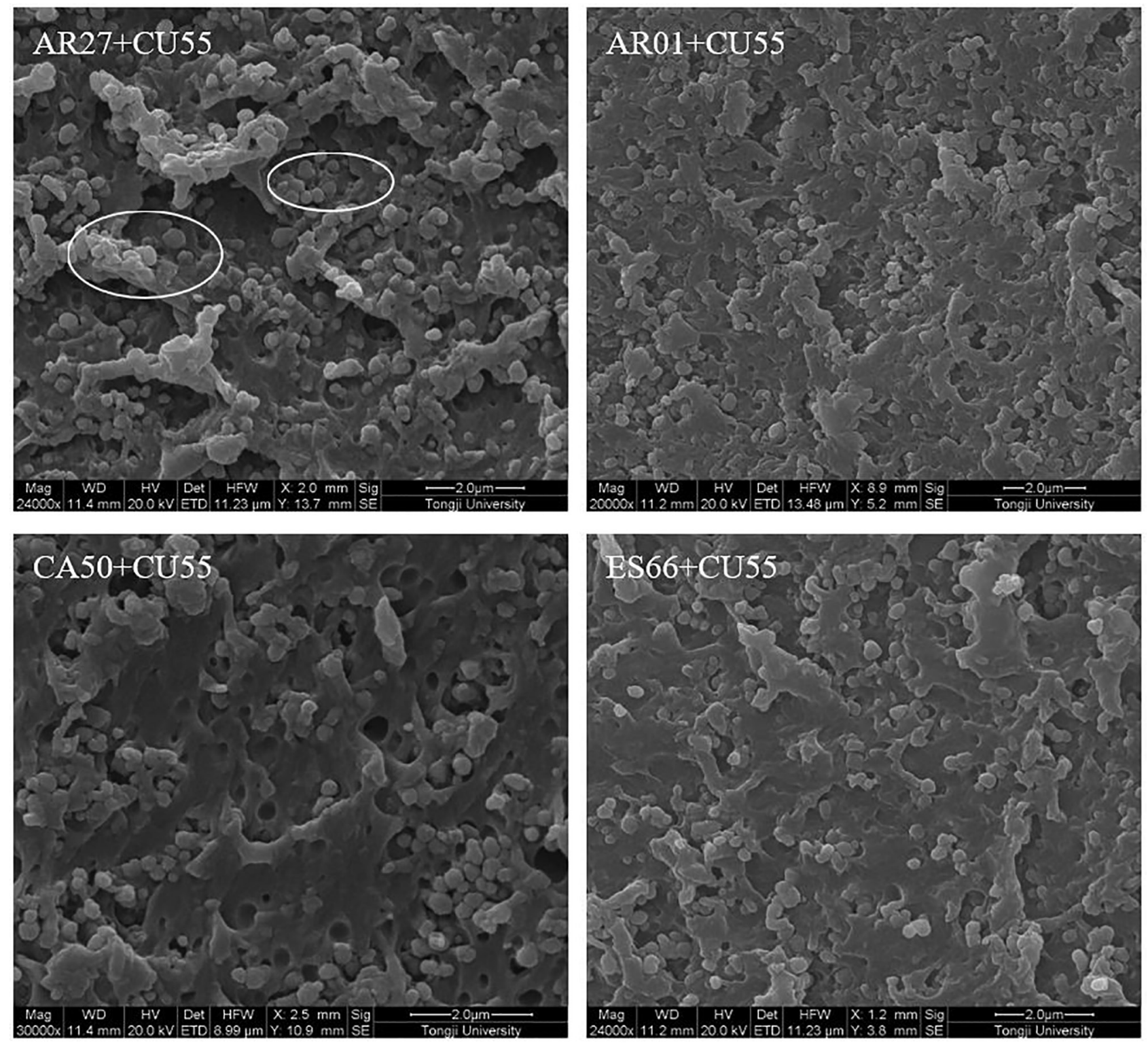

Figure 4: SEM image of micro morphology inside $2 \mathrm{~K}$ WPU coating based on different resins.

salt spray resistance. Obviously, average rust width along two scribe marks on the coating based on AR01 was larger. Hence, polyacrylic polyol exhibited less barrier to rust accumulation and spreading.

The result was consistent with the prediction according to the hydroxyl content of resins and crosslinking degree of coatings. The $\mathrm{OH} / \mathrm{CH}$ ratio of polyester polyol ES66, polycarbonate polyol CA50, and polyacrylic polyol AR01 are 1.21, 1.01, and 0.92, respectively. And crosslinking degrees of coatings based on ES66, CA50, and AR01 are 75\%, 74\%, and $73 \%$, respectively. Accordingly, ES66 and CA50 with high hydroxyl content tended to form a compact PU film with high crosslinking degree, and showed perfect salt spray resistance. Moreover, the strong cohesive energy brought by ester group in ES66 and CA50 is beneficial to the formation of compact and tight PU film and the improvement of salt spray resistance.

\subsection{Effect of resins on the wet adhesion}

\subsubsection{Effect of hydroxyl content in resins on the wet adhesion}

The wet adhesion of $2 \mathrm{~K}$ WPU coating based on polyacrylic polyols with different hydroxyl content were presented in Figure 6a: AR01 > AR70 > AR46 > AR42 > AR27. 
A

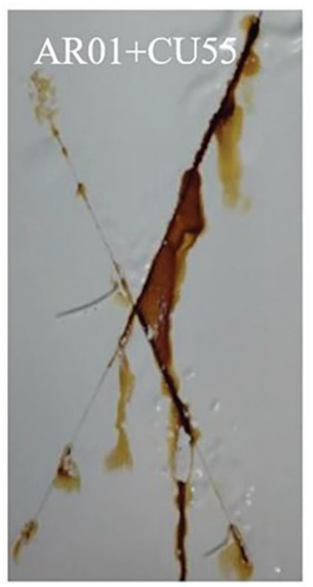

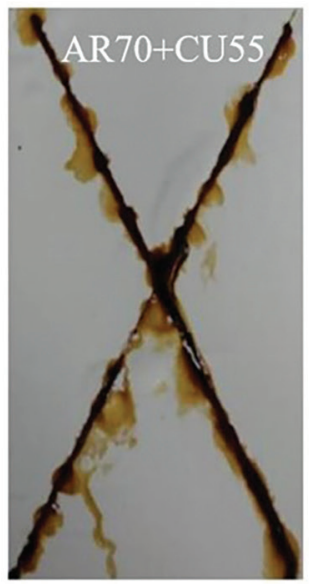
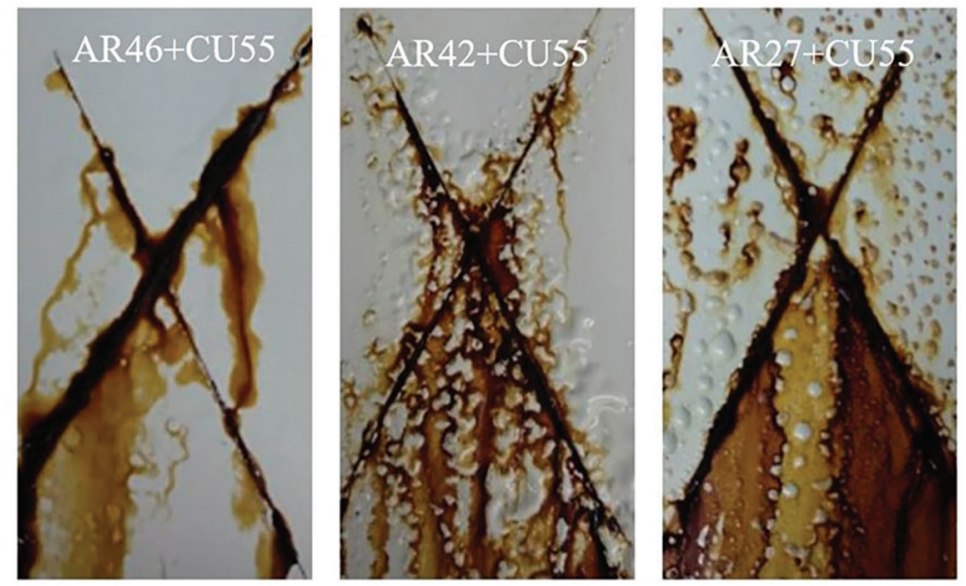

B
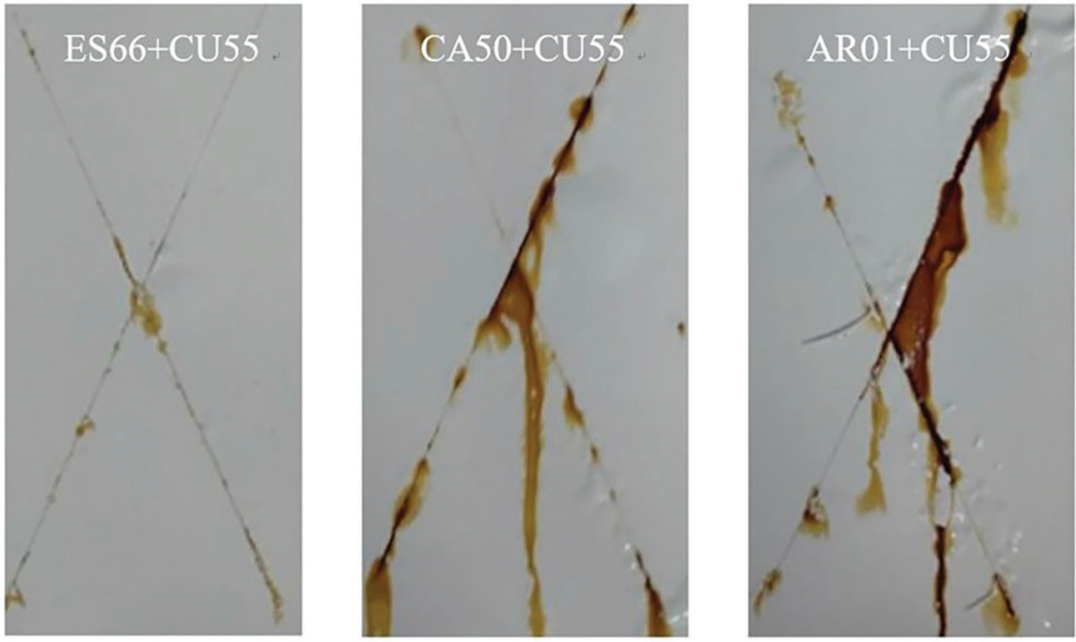

Figure 5: Images of 2K WPU coating based on polyacrylic polyols with different hydroxyl content (a) and different resins (b) after salt spray for $600 \mathrm{~h}$.

This sequence of coatings based on polyacrylic polyols was consistent with salt spray resistance, which means that wet adhesion is the key property to realize its salt spray resistance for steel panels with $\mathrm{x}$-cut paints. And the wet adhesion of $2 \mathrm{~K}$ WPU coating always decreases before rusting during salt spray.

Good agreement was found between hydroxyl content in resins, crosslinking degree, and wet adhesion of $2 \mathrm{~K}$ WPU coating. Resins with more $-\mathrm{OH}$ tended to react with -NCO provided by polyisocyanurate to form PU film with higher crosslinking degree. During immersion in water, $\mathrm{H}_{2} \mathrm{O}$ molecules can diffuse into the interface via some micro defects because some pigment particles cannot be wrapped by enough PU in coatings based on the low hydroxyl content resins such as AR27. And the accumulation of $\mathrm{H}_{2} \mathrm{O}$ at the interface would form a lateral pressure to weaken the adhesion of coatings. Hence, the compact and tight coatings based on the resin with more hydroxyl groups tend to show more excellent water resistance. AR01 is the best case on the hydroxyl content and wet adhesion of coatings among these polyacrylic polyols. The wet adhesion of 2K WPU coating based on AR01 with the $\mathrm{OH} / \mathrm{CH}$ of 0.92 was graded as level 3 even if the sample was immersed in water at $40^{\circ} \mathrm{C}$ for $8 \mathrm{~h}$.

\subsubsection{Effect of different types of resins on the wet adhesion}

The wet adhesion of $2 \mathrm{~K}$ WPU coating on the substrate for different resins after immersed in water for several hours was presented in Figure 6b: AR01 > ES66 > CA50. And it is easier for the wet adhesion of coatings based on polyester polyol ES66 and polycarbonate polyol CA50 to deteriorate from level 0 to level 5 than AR01.

Obviously, ES66 and CA50 have a worse wet adhesion than AR01 even though they have more - $\mathrm{OH}$, which proves 

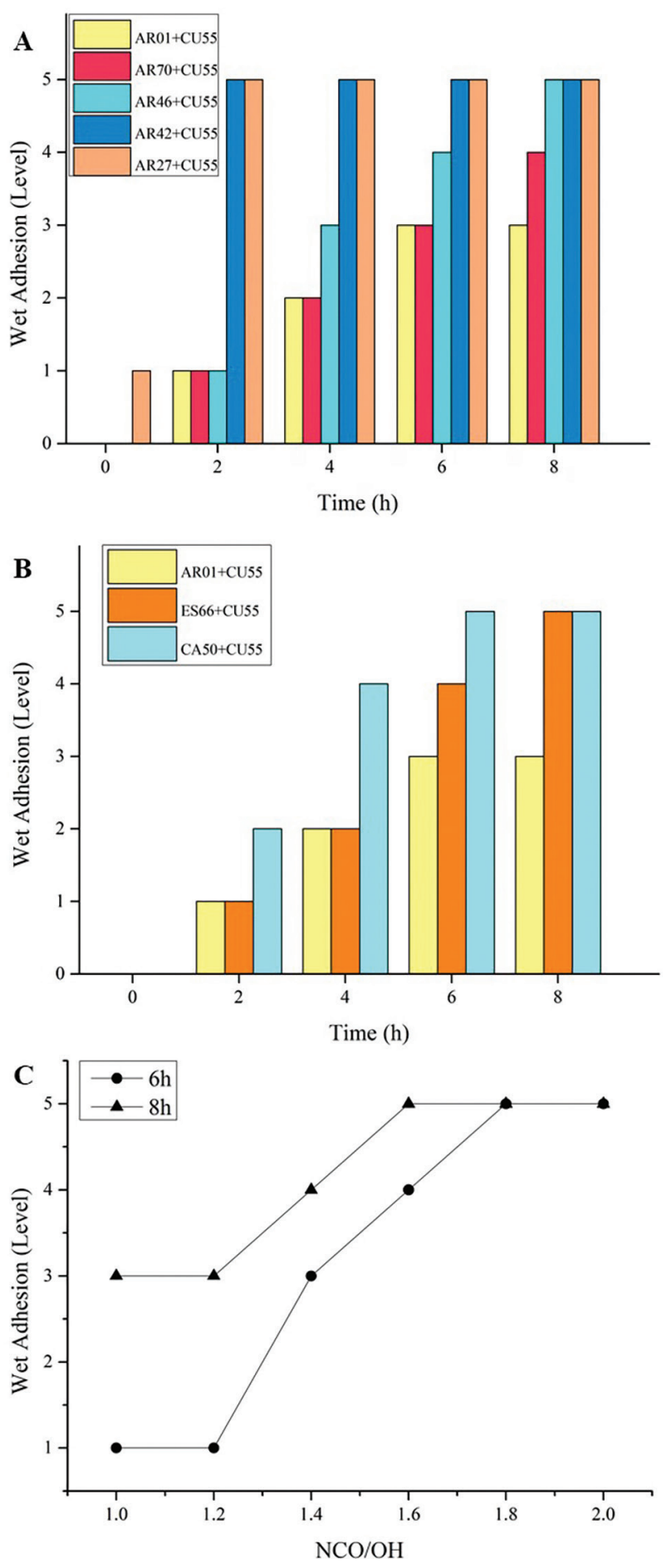

Figure 6: The wet adhesion of $2 \mathrm{~K}$ WPU coating based on polyacrylic polyols with different hydroxyl content (a), different resins (b), and different $\mathrm{NCO} / \mathrm{OH}$ (c).

the weakness of polyester or polycarbonate polyol on resistance to water even though its salt spray resistance and hydroxyl content was best of them. The wet adhesion is a key property to realize its salt spray resistance of coating, but not the only one factor to decide its performance on salt spray. Because the ester group in these resins is easy to hydrolyze in warm water, PU macromolecular main chains are easy to break accordingly, and then the cohesion and wet adhesion of the PU film wrapping pigment particles will be weakened.

\subsubsection{Effect of $\mathrm{NCO} / \mathrm{OH}$ on the wet adhesion}

In practical use, excess polyisocyanurate would be added into coatings so as to consume enough hydroxyl groups in resins because of too much water in $2 \mathrm{~K}$ WPU coating. According to the reaction mechanism in theory between $-\mathrm{OH}$ and $-\mathrm{NCO}$ and some engineering practice, the effect of $\mathrm{NCO} / \mathrm{OH}$ ranging from 1.0 to 2.0 on wet adhesion of coatings was studied in this paper. Figure $6 \mathrm{c}$ presents the wet adhesion of $2 \mathrm{~K}$ WPU coating with different $\mathrm{NCO} / \mathrm{OH}$. The wet adhesion of coatings cannot be improved by excess - NCO, and it deteriorated from level 1 or 3 to level 5 with the increase of $\mathrm{NCO} / \mathrm{OH}$ after immersed in the water at $40^{\circ} \mathrm{C}$ for $6 \mathrm{~h}$ or $8 \mathrm{~h}$.

There are two reasons for effect of $\mathrm{NCO} / \mathrm{OH}$ on the wet adhesion. On the one hand, the decrease of hydroxyl content in coatings is the main cause for the deterioration of wet adhesion. In general, the adhesion was regarded as a linkage between hydroxyl group of coatings and hydroxyl group of substrates. Addition of - $\mathrm{NCO}$ can be mainly used to react with - $\mathrm{OH}$ of resin in the first stage, and the rest of - $\mathrm{OH}$ has decreased with the increase of $\mathrm{NCO} / \mathrm{OH}$ accordingly. As a result, there is less and less - $\mathrm{OH}$ in $2 \mathrm{~K}$ WPU coating with the increase of $\mathrm{NCO} / \mathrm{OH}$ to link with other groups of metal substrates. On the other hand, excess - $\mathrm{NCO}$ can react with $\mathrm{H}_{2} \mathrm{O}$ to generate $\mathrm{CO}_{2}$ and urea without $\mathrm{C}-\mathrm{O}$, which was proved by the increase of $\mathrm{C}=\mathrm{O} / \mathrm{C}-\mathrm{O}$ in Figure 2 . And cracked physical bubbles or other micro defects inside the $\mathrm{PU}$ film caused by $\mathrm{CO}_{2}$ in the reaction between - $\mathrm{NCO}$ and $\mathrm{H}_{2} \mathrm{O}$ would form a path for water to permeate into the interface. Furthermore, the accumulation of $\mathrm{H}_{2} \mathrm{O}$ at the interface would form a lateral pressure to weaken the adhesion of coatings. Therefore, excess - NCO will bring about the deterioration of wet adhesion of $2 \mathrm{~K}$ WPU coating.

\section{Conclusions}

In this study, the effect of resins on salt spray resistance and wet adhesion of $2 \mathrm{~K}$ WPU coating was studied to further provide some theory foundation for selecting resins and optimization of $\mathrm{NCO} / \mathrm{OH}$ to improve anticorrosive 
performance of coatings. The results could be concluded as follows.

(1) Resins with more $-\mathrm{OH}$ tend to react with polyisocyanurate to form a compact PU film with higher crosslinking degree, which leads to the better wet adhesion and salt spray resistance. The sequence of hydroxyl content in polyacrylic polyols was consistent with their crosslinking degree, salt spray resistance and wet adhesion of coatings.

(2) For commonly used resins, polyester polyol and polycarbonate polyol had better performance than polyacrylic polyol on salt spray resistance due to more hydroxyl and ester groups in resins. The wet adhesion of coatings based on polyester and polycarbonate polyol decreased from level 1 to level 5 with the increase of soak time because of the easily hydrolyzed ester group in resins.

(3) The wet adhesion of $2 \mathrm{~K}$ WPU coating would decrease from level 1 to level 5 with the increase of $\mathrm{NCO} / \mathrm{OH}$. Because there are less - $\mathrm{OH}$ in coatings to link with substrates with the increase of $\mathrm{NCO} / \mathrm{OH}$. Moreover, cracked physical bubbles inside the film caused by $\mathrm{CO}_{2}$ generated by the reaction between -NCO and $\mathrm{H}_{2} \mathrm{O}$ would form a path for water to permeate into the interface, which weaken the adhesion of coatings.

\section{Acknowledgments}

Thanks to the company Covestro Polymers (China) Co., Ltd. and the employees for supporting this work. This study was finished during the internship in Covestro.

\section{References}

1. Sørensen P.A., Kiil S., Dam-Johansen K., Weinell C.E., Anticorrosive coatings: a review. J. Coat. Technol. Res., 2009, 6, 135-176.

2. Yan J., Shi J.J., Zhang P.G., Tian W.B., Zhang Y.M., Preparation and properties of epoxy/basalt flakes anticorrosive coatings. Mater. Corros., 2018, 69, 1-7.

3. Armelin E., Pla R., Liesa F., Ramis X., Iribarren J.I., Aleman C., Corrosion protection with polyaniline and polypyrrole as anticorrosive additives for epoxy paint. Corros. Sci., 2008, 50, 721-728.
4. Hofland A., Alkyd resins: from down and out to alive and kicking. Prog. Org. Coat., 2012, 73, 274-282.

5. Noreen A., Zia K.M., Zuber M., Tabasum S., Saif M.J., Recent trends in environmentally friendly water-borne polyurethane coatings: a review. Korean J. Chem. Eng., 2016, 33, 388-400.

6. Xu F., Qian B.R., Hu Z., Chen W.D., Zhuang Z.Y., Zhu B.Y., et al., A novel route to emulsifier-free, waterborne hydroxyl functional polyacrylate with low VOC level and its application in 2K-WPU coatings. J. Macromol. Sci. A., 2013, 50, 555-561.

7. Huang H.Y., Huang T.C., Lin J.C., Chang J.H., Lee Y.T., Yeh J.M., Advanced environmentally friendly coatings prepared from amine-capped aniline trimer-based waterborne electroactive polyurethane. Mater. Chem. Phys., 2013, 137, 772-780.

8. Kiosidou E.D., Karantonis A., Pantelis D.I., Silva E.R., Bordado J.C.M., Rust morphology characterization of polyurethane and acrylic-based marine antifouling paints after salt spray test on scribed specimens. J. Coat. Technol. Res., 2017, 14, 1381-1395.

9. Huang M.X., Yang J.L., Salt spray and EIS studies on HDI microcapsule-based self-healing anticorrosive coatings. Prog. Org. Coat., 2014, 77, 168-175.

10. Miyauchi K., Takita Y., Yamabe H., Yuasa M., A study of adhesion on stainless steel in an epoxy/dicyandiamide coating system: influence of glass transition temperature on wet adhesion. Prog. Org. Coat., 2016, 99, 302-307.

11. Glass P., Chung H., Washburn N.R., Sitti M., Enhanced wet adhesion and shear of elastomeric micro-fiber arrays with mushroom tip geometry and a photopolymerized p(DMA-Co-MEA) tip coating. Langmuir, 2010, 26, 17357-17362.

12. Wu T.H., Foyet A., Kodentsov A., van der Ven L.G.J., van Benthem R.A.T.M., de With G., Wet adhesion of epoxy-amine coatings on 2024-T3 aluminum alloy. Mater. Chem. Phys., 2014, 145, 342-349.

13. Meis N.N.A.H., van der Ven L.G.J., van Benthem R.A.T.M., de With G., Extreme wet adhesion of a novel epoxy-amine coating on aluminum alloy 2024-T3. Prog. Org. Coat., 2014, 77, 176-183.

14. Akbarian M., Olya M.E., Mahdavian M., Ataeefard M., Effects of nanoparticulate silver on the corrosion protection performance of polyurethane coatings on mild steel in sodium chloride solution. Prog. Org. Coat., 2014, 77, 1233-1240.

15. Cai K.W., Zuo S.X., Luo S.P., Yao C., Liu W.J., Ma J.F., et al., Preparation of polyaniline/graphene composites with excellent anti-corrosion properties and their application in waterborne polyurethane anticorrosive coatings. RSC Adv., 2016, 6, 95965-95972.

16. Rashvand M., Ranjbar Z., Effect of nano-ZnO particles on the corrosion resistance of polyurethane-based waterborne coatings immersed in sodium chloride solution via EIS technique. Prog. Org. Coat., 2017, 76, 1413-1417.

17. Li J., Cui J.C., Yang J.Y., Li Y.Y., Qiu H.X., Yang J.H., Reinforcement of graphene and its derivatives on the anticorrosive properties of waterborne polyurethane coatings. Compos. Sci. Technol., 2016, $129,30-37$ 